\title{
Laser anemometer measurements of trailing vortices in water
}

\author{
By G. R. BAKER, S. J. BARKER, K. K. BOFAH \\ AND P. G. SAFFMAN \\ Graduate Aeronautical Laboratories, California Institute of Technology, Pasadena
}

(Received 10 October 1973)

A series of measurements of trailing vortices behind lifting hydrofoils is described. These measurements were made in the Caltech Free-Surface Water Tunnel, using a laser-Doppler velocimeter to measure two components of velocity in the vortex wake. Two different model planforms were tested, and measurements were made at several free-stream velocities and angles of attack for each. Velocity profiles were measured at distances downstream of the model of from five to sixty chord lengths. These measurements are the first results of a continuing experimental programme.

In $\S 3$ of this paper, the theory of trailing vortices is discussed. The effects of 'vortex wandering' upon the measurements are computed, and the corrected results are seen to be in reasonable agreement with the theory.

\section{Introduction}

There have been numerous recent attempts to measure velocities in trailing vortices, stimulated by interest in the problem of aircraft wake turbulence. Several types of measurements have been made to date. There have been freeflight measurements (Caiger \& Gould 1971; Chevalier 1973), in which one aircraft follows another to measure velocities in its wake. These have not yielded accurate results because of the difficulty in locating the vortex cores. There have been wind-tunnel measurements of stagnation pressure (Mason \& Marchman 1972), but these are subject to doubt because of probe interference effects. It is questionable whether any material probe can be placed in the core of a small trailing vortex without significantly disturbing the flow. There have also been measurements in tow basins of velocities in the unsteady decaying vortices (Miller \& Brown 1971; Lezius 1973). In this case the flow is time dependent, which makes the mean velocity profiles in the vortices difficult to determine.

The only existing measurement technique which can guarantee no disturbance of the flow with high spatial resolution is laser-Doppler anemometry. Recent laser-Doppler velocimeter (LDV) measurements of trailing vortices in a wind tunnel have been reported by Orloff \& Grant (1973). In the present experimental programme at Caltech, an LDV is being used to measure axial and tangential velocity profiles in trailing vortices in a water tunnel. The water tunnel has the advantages of high Reynolds numbers at relatively low speeds, 


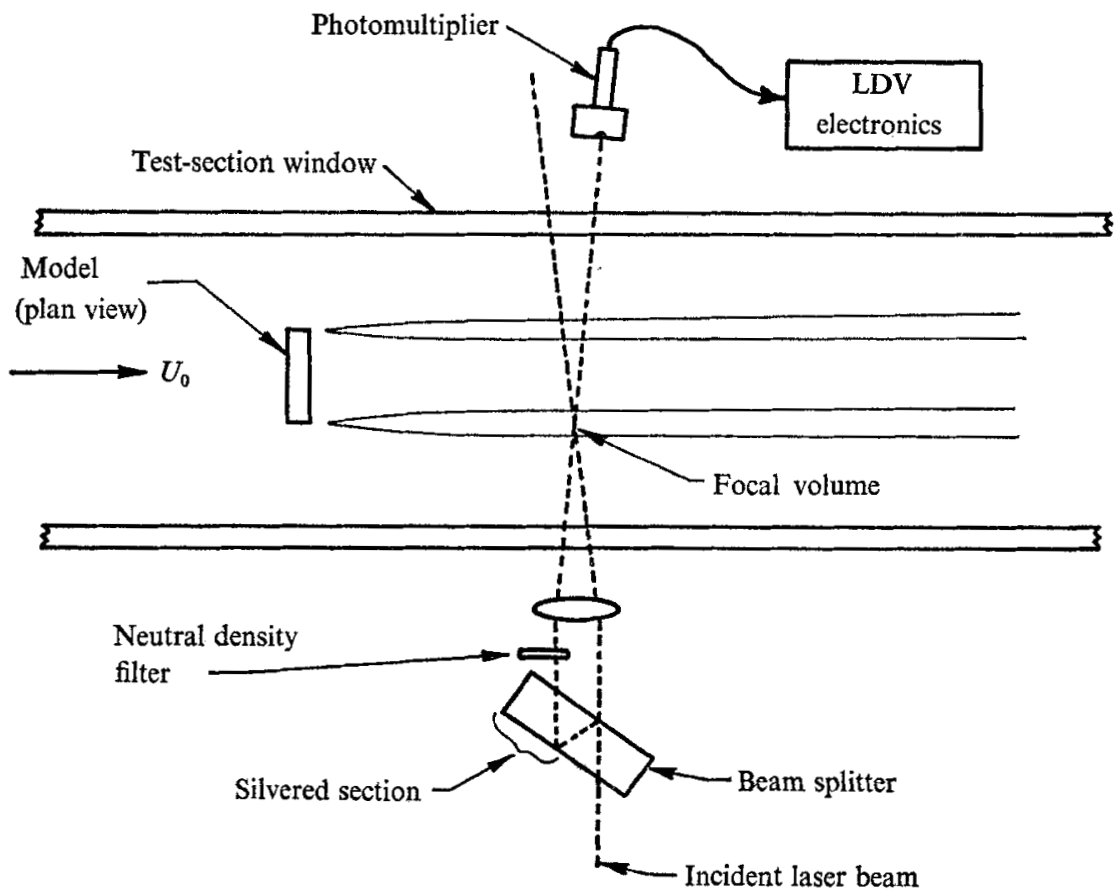

Figure 1. Experimental geometry.

and the presence of sufficient light-scattering particles in the water to produce a nearly continuous Doppler signal without 'seeding' the flow with foreign particles. The ease of flow visualization in water makes it possible to measure photographically the position of the vortex and its degree of 'wandering', which will be diseussed in $\S 3$.

\section{Experimental programme}

\subsection{Test facility}

The Caltech Free-Surface Water Tunnel has a test section which is $51 \times 51 \mathrm{~cm}$ in cross-section and $244 \mathrm{~cm}$ long. The maximum flow velocity is $730 \mathrm{~cm} / \mathrm{s}$. Velocities between 200 and $300 \mathrm{~cm} / \mathrm{s}$ correspond to Froude numbers near one and therefore are not usable. The free-stream turbulence level of the tunnel is about $0.5 \%$. For a complete description of this facility, see Knapp \& Levy (1948).

The two hydrofoil models used in this study have a span of $15 \cdot 2 \mathrm{~cm}$ and a chord of $2.46 \mathrm{~cm}$. Both have a symmetric semicircular arc profile, with a maximum thickness of $0.35 \mathrm{~cm}$ and a leading-edge radius of $0.08 \mathrm{~cm}$. One of the models has a rectangular planform and the other has a planform with semicircular tips. Both models are mounted horizontally in the tunnel section by means of a thin vertical support strut attached to the centre of the span of the model. Above the water surface, the vertical strut is attached to a mechanism which controls the angle of attack and depth of the model. The model is located near the upstream end of the test section so that measurements can be made at distances of up to sixty chord lengths downstream. (See figure 1.) 


\subsection{Laser-Doppler instrumentation}

Use of a laser-Doppler velocimeter (LDV) has several important advantages over conventional techniques in this experiment. Perhaps the most significant is the fact that there is no material probe in the fluid flow, and hence no flow interference. Flow visualization studies suggest that trailing vortices are extremely sensitive to disturbances created by even very small probes. Other advantages of the LDV are its linear response to velocity, its small measuring volume and the ability to measure one velocity component independently of the others.

The LDV used in this experiment is mounted on a traverse which moves the entire optical system with respect to the water tunnel. This LDV operates in the 'local oscillator' mode, whose principle will be described here very briefly. The beam from a $5 \mathrm{~mW}$ helium-neon laser is split into two parallel beams by a glass-prism beam splitter which uses partial internal reflexion of the incident beam. One of the beams emerging from the prism is weaker than the other, and this beam is further attenuated by a factor of 100 with a neutral density filter. Both beams then pass through a biconvex lens of focal length $30 \mathrm{~cm}$. The two beams cross at a point within the fluid flow as they pass through the test section. On the other side of the tunnel, the weaker beam passes through an aperture $1 \mathrm{~mm}$ in diameter and enters a photomultiplier tube. Here the light from the weak 'reference' beam is mixed with light from the brighter beam which has been scattered from the volume in which the two beams intersect. This scattered light is Doppler shifted by the motion of the scattering particles, which are assumed to move with the local fluid velocity. Thus the scattered light has a slightly different frequency from the reference light and the combination of the two produces a beat frequency in the photomultiplier tube. This beat frequency is directly proportional to one component of the fluid velocity. For a more thorough explanation of the LDV principle, see Goldstein (1967), Adrian (1972) or Wang (1972).

The beat frequency from the photomultiplier is amplified and band-pass filtered to remove noise outside the frequency range of interest. The signal is then further amplified and clipped to eliminate most of the random amplitude modulation of the raw Doppler signal. The clipped signal is fed into a phaselocked loop, which produces a continuous square wave of the same frequency as the Doppler signal. The phase-locked loop reduces the effect of momentary signal dropouts which are caused by fluctuations in the number of scattering particles in the focal volume. The phase-locked loop can be used in the LDV system as long as the turbulence level does not exceed $12 \%$. At higher turbulence levels, it cannot accurately track the fluctuations of the Doppler frequency.

The square-wave output of the phase-locked loop is fed into a digital counter which averages the Doppler frequency over a 10 s period. The counter frequency is then converted into a velocity averaged over the same period. The squarewave signal also goes to the input of a frequency-to-voltage converter (Anadex model PI-408R). This device produces an analog voltage proportional to the 
Doppler frequency and thus to the velocity. The converter averages the frequency over about fifty periods, so that the resulting instantaneous velocity signal has a bandwidth of from 0 to $\frac{1}{50}$ of the Doppler frequency. The velocity signal is fed into an r.m.s. voltmeter to measure the turbulence intensity.

The accuracy of any LDV system is limited by what is known as 'ambiguity noise' (George 1972). This is a broadening of the Doppler signal spectrum, or noise on the demodulated velocity signal, which has three causes: (i) mean velocity gradients across the LDV focal volume, (ii) turbulent fluctuations within the focal volume and (iii) the finite time of transit of scattering particles passing through the focal volume. Ambiguity noise limits the accuracy of measurements of velocity fluctuations, but has no effect upon mean velocity measurements. For the focal volume used in the present experiment, which is approximately $1 \times 0.05 \times 0.05 \mathrm{~mm}$, the noise produced by (ii) is negligible. Noise from (i) may be significant in the high shear region in the core of the vortex, but a more important cause of measured velocity fluctuations in this region is the 'vortex wandering', which is discussed below. Noise from (iii) will be the same in laminar as in turbulent flow for a given focal volume, and has been measured in the laboratory. This component of the ambiguity noise is equivalent to a turbulence level of $0.25 \%$, which is thus the resolution limit of the system for velocity fluctuations.

\subsection{Experimental results}

The present series of measurements included axial and tangential velocity profiles in the vortex wakes behind two different model hydrofoils at distances of from five to sixty chord lengths downstream. The axial velocity component was measured directly by aligning the LDV with the free-stream flow. To obtain the tangential component, the velocity component at a $45^{\circ}$ angle to the free stream was measured with the LDV, and this was used together with the axial velocity to compute the tangential velocity. $\dagger$ Velocity traverses at each downstream station were made in the horizontal plane, starting well out in the free stream and proceeding inwards to a point past the centre of the span of the model. Each mean velocity measurement represents a 30 s average, which was found to give extremely repeatable results.

Figure 2 shows typical velocity data for the model with square tips. Shown on this figure are axial and tangential velocity profiles for two values of $x / c$ (distance downstream divided by chord length), and profiles of the axial fluctuations. Flow visualization studies suggest that these measured velocity fluctuations may be caused primarily by the random motion or wandering of the trailing vortex about the measurement point. The velocity gradients near the core of the vortex are high, so that the relatively small motions of the vortex can produce large velocity fluctuations. The degree of vortex wandering and its effect upon the measured velocities will be discussed in $\S 3$.

$\dagger$ The tangential component could not be measured directly by aligning the LDV at $90^{\circ}$ to the free-stream flow, because the mean tangential velocity passes through zero at the centre of the vortex. The LDV measures the magnitude but not the sign of the velocity, so that velocities with near zero mean are very difficult to measure unless frequency biasing is used. 

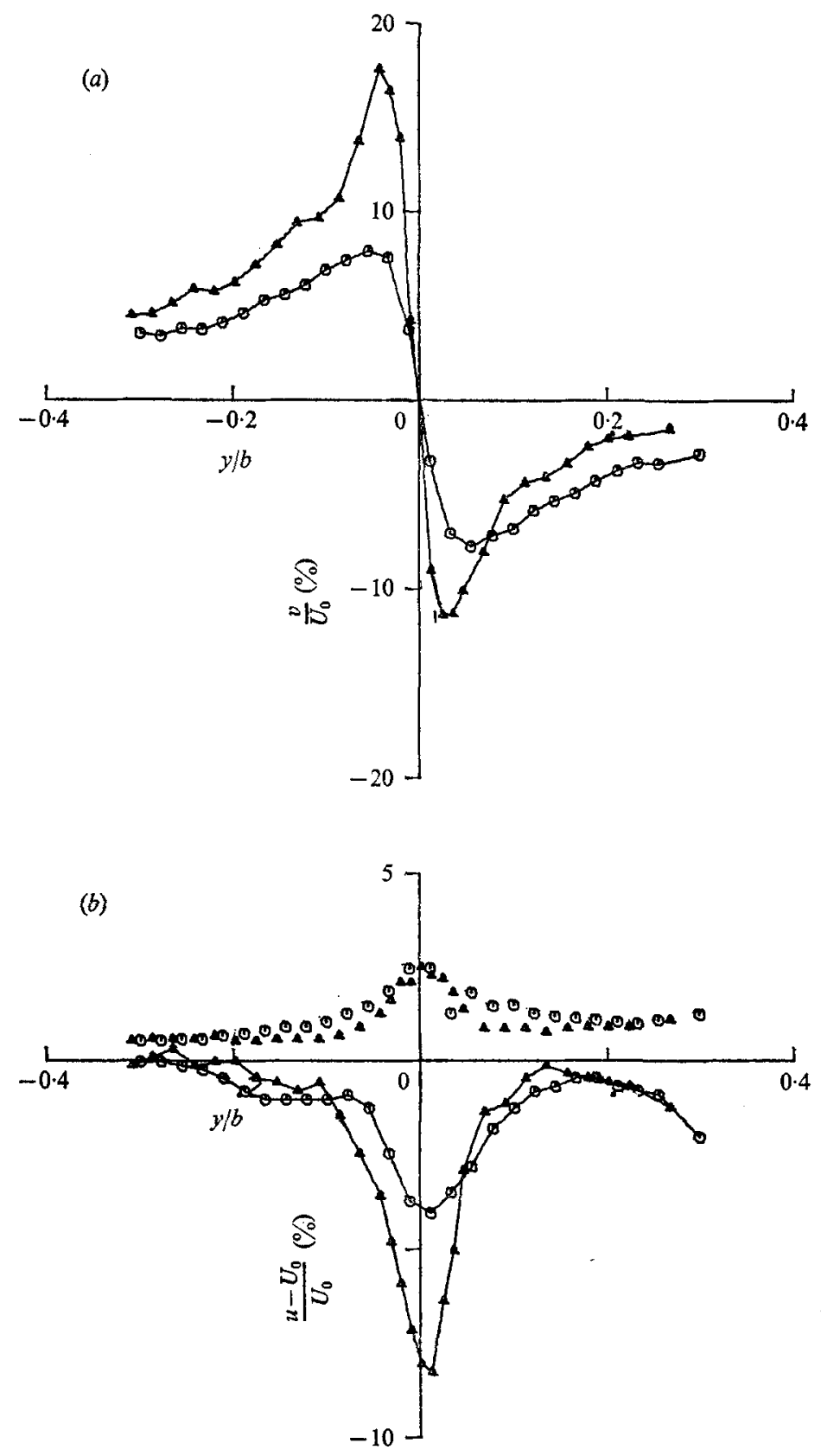

FIGURE 2. Velocity profiles in trailing vortex. $R_{\mathrm{c}}=22000, U_{0}=90 \mathrm{~cm} / \mathrm{s}, \alpha=10^{\circ}$, aspect ratio $=6 \cdot 18$, wing span $b=15 \cdot 24 \mathrm{~cm} . \Lambda, x / c=10 ; \boldsymbol{Q}, x / c=20$. (a) Tangential velocity. (b) Axial velocity defect (lower points) and fluctuations (upper points).

The repeatability and small scatter of the data in this experiment show that the use of the LDV in a water tunnel is a practical means of measuring velocities in trailing vortices. The next step in this experimental programme will be to find to what extent the vortex wandering phenomenon is caused by free-stream 
turbulence. This will be done by reducing the turbulence level of the water tunnel below its present value of $0.5 \%$ with the use of additional flow straighteners.

\section{Theory and analysis}

\subsection{Trailing vortex theory}

We now compare the experimental data with the available theory. Moore \& Saffman (1973, hereafter referred to as I) have recently given a theory for the structure of laminar trailing vortices. For the case where the wing loading varies like the square root of the distance from the wing tip, the tangential velocity near the centre of the vortex is given by

$$
v(r, x)=\beta \Gamma\left(\frac{5}{4}\right) r\left(4 \nu x / U_{0}\right)^{-\frac{3}{4}} M\left(\frac{3}{4} ; 2 ;-U_{0} r^{2} / 4 \nu x\right) .
$$

Here $r$ is the radial distance from the axis of the vortex, $x$ is the distance downstream of the wing, $v$ is the kinematic viscosity, $U_{0}$ is the free-stream velocity or speed of the wing and $M$ is the confluent hypergeometric function (Abramovitz $\&$ Stegun 1965, p. 503). The quantity $\beta$ is related to the wing loading and mechanism of roll-up. We shall use the estimate, which incorporates a correction for finite aspect ratio exact for elliptic loading,

$$
\beta=\frac{6^{\frac{1}{2}}}{2} U_{0} \alpha \frac{c}{b^{\frac{1}{2}}}\left(1+\frac{\pi c}{2 b}\right)^{-1}
$$

with $\alpha$ measured in radians, where $c$ is the chord length and $b$ the span. This value corresponds to an elliptically loaded thin wing, the root section having a lift coefficient of $2 \pi \alpha$, with the 'contraction factor' $\lambda$ set equal to $1 \cdot 5$. (See $I$ for further details, but note that a larger value of $\beta$ was used there for comparison with Olsen's (1971) towing-tank data, corresponding to the solution of the lifting line theory equation for a semi-infinite rectangular wing. The present experiments indicate that (2) is a better estimate, as should be the case for an aspect ratio of 6 .)

The radius $r_{1}$ of the vortex core is defined as the value of $r$ for which $v$ is a maximum:

$$
r_{1}=2 \cdot 92\left(v x / U_{0}\right)^{\frac{1}{2}}
$$

The maximum tangential velocity $v_{1}$ is given by

$$
v_{1}=0 \cdot 49 \beta\left(v x / U_{0}\right)^{-\frac{1}{4}} .
$$

The axial vorticity $\xi$ on the axis is $2 \partial v / \partial r$ evaluated $r=0$, and is given by

$$
\xi=\frac{\Gamma\left(\frac{5}{4}\right)}{2^{\frac{1}{2}}} \beta\left(\frac{\nu x}{U_{0}}\right)^{-\frac{3}{4}}
$$

The axial velocity $u(r, x)$, measured relative to the free stream, is the sum of two terms. The first term $u_{v}$ is due to the pressure field induced by the roll-up and 
decay of each trailing vortex. The second term $u_{\delta}$ is a velocity defect due to retardation in the boundary layer (of thickness $\delta$ ) on the wing. Thus,

$$
\begin{aligned}
u(r, x) & =u_{v}+u_{\delta} \\
& =\frac{\beta^{2}}{U_{0}}\left(\frac{\nu x}{U_{0}}\right)^{-\frac{1}{2}} W_{\frac{1}{2}}-0 \cdot 21 U_{0} \delta\left(\frac{v x}{U_{0}}\right)^{-\frac{1}{2}} M\left(\frac{1}{2} ; 1 ; \frac{-U_{0} r^{2}}{4 v x}\right) .
\end{aligned}
$$

$W_{\frac{1}{2}}$ is a function of $U_{0} r^{2} / 4 v x$. It can be expressed as an integral of confluent hypergeometric functions; a plot is given in I, figure $3(a)$. Note that $W_{\frac{1}{2}}(0)=-0 \cdot 13$, $W_{1}$ is positive for $r>1 \cdot 4\left(\nu x / U_{0}\right)^{\frac{1}{2}}$ and $W_{1} \sim\left(\nu x / U_{0} r^{2}\right)^{\frac{1}{2}}$ for large values of the argument. The flux deficit per unit span in the boundary layer is $U_{0} \delta$. The above expression is for a rectangular wing, for which $\delta$ is assumed independent of the spanwise station. In I it was assumed that $\delta$ is given by the momentum thickness $\delta_{2}$ of a Blasius boundary layer, i.e. $\delta_{2}=1 \cdot 33\left(\nu c / U_{0}\right)^{\frac{1}{2}}$. However, arguments were given [see I, paragraph following equation (3.29)] that the displacement thickness might be more appropriate for the axial velocity on the axis of the vortex, i.e. $\delta_{1}=3 \cdot 44\left(v c / U_{0}\right)^{\frac{1}{2}}$. The velocity on the axis is written as $\Delta U$, where

$$
\Delta U=\Delta U_{v}+\Delta U_{\delta}=-0 \cdot 13 \frac{\beta^{2}}{U_{0}}\left(\frac{U_{0}}{\nu x}\right)^{\frac{1}{2}}-U_{0} \chi\left(\frac{c}{x}\right)^{\frac{1}{2}} .
$$

Here $\chi=0 \cdot 28$ or $0 \cdot 72$ depending on whether the momentum or displacement thickness is used in estimating the boundary-layer retardation.

\subsection{Vortex wandering}

These predictions cannot be compared directly with experiment because the vortex is observed to wander in a random manner. The measured profiles are time averages at positions fixed relative to the wing, and are therefore weighted averages of the instantaneous profiles (1) and (6). It is believed that the vortex wandering is due mainly to free-stream turbulence, $\uparrow$ so that the predictions can be corrected for the purpose of comparison with the experimental data.

We expect the turbulence to be equivalent to an eddy diffusivity $\kappa$. Then the axis of the vortex will fluctuate randomly about its mean position in a transverse plane at given $x$ with a probability density

where

$$
\begin{gathered}
p(\eta, \zeta)=\left(1 / 2 \pi \sigma^{2}\right) \exp \left[-\left(\eta^{2}+\zeta^{2}\right) / 2 \sigma^{2}\right] \\
\sigma^{2}=2 \kappa x / U_{0} .
\end{gathered}
$$

We have determined $\kappa$ in an approximate manner by enlarging photographs of a vortex marked with dye and measuring the mean-square displacement of the axis. There is considerable scatter, but this procedure gave values for the ratio $\nu / \kappa$ of about 0.4 for $U_{0}=30 \mathrm{~cm} / \mathrm{s}$ and about 0.2 for $U_{0}=90 \mathrm{~cm} / \mathrm{s}$.

We assume that the theoretical profiles (1) and (6) are valid instantaneously relative to the instantaneous position of the axis. Then observed quantities are

The same degree of wandering was observed for a stream of dye released from the model in a zero-lift configuration. Preliminary measurements with reduced free-stream turbulence showed significantly less vortex wandering. Far downstream, some wandering may be due to the mutual instability of the two trailing vortices, but estimates of this effect suggest that it is negligible for the present values of $x / c$. 


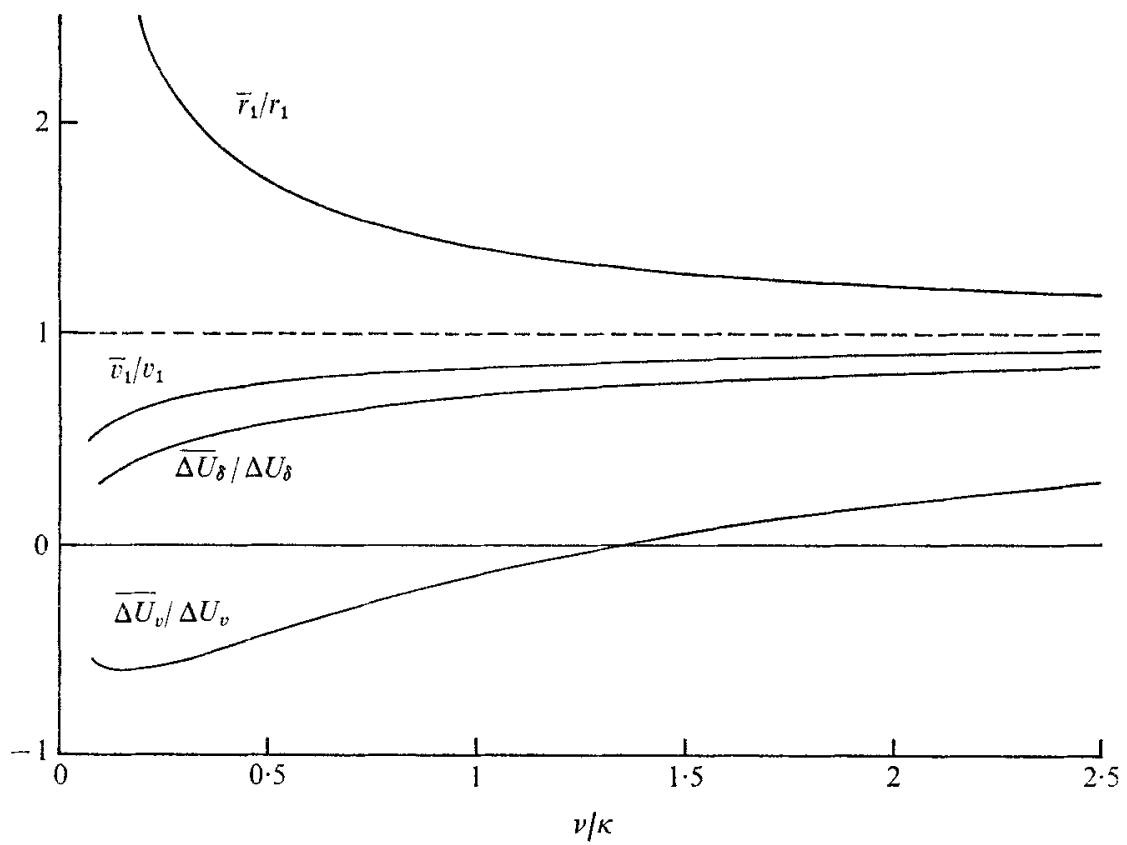

FraURE 3. Effect of vortex wandering on core radius, maximum tangential velocity, and axial velocity on the mean axis.

averages, denoted by overbars, over the wandering of the vortex axis. Thus for the axial velocity

$$
\ddot{u}(r, x)=\iint u\left\{\left[(r-\eta)^{2}+\zeta^{2}\right]^{\frac{1}{2}}, x\right\} p(\eta, \zeta) d \eta d \zeta
$$

and for the tangential velocity

$$
\bar{v}(r, x)=\iint \frac{\eta-r}{\left[(r-\eta)^{2}+\zeta^{2}\right]^{\frac{1}{2}}} v\left\{\left[(r-\eta)^{2}+\zeta^{2}\right]^{\frac{1}{2}}, x\right\} p(\eta, \zeta) d \eta d \zeta,
$$

where $r$ in these expressions is measured from the mean position of the axis. The extra term in (11) arises from the need to incorporate a geometrical factor.

Substituting for $p(\eta, \zeta)$ from (8) and for $u$ and $v$ from (6) and (1), the integrals can be reduced to single integrals involving modified Bessel functions and confluent hypergeometric functions, which can be put into dimensionless form as functions of $x / c, U_{0} r^{2} / \nu x$ and $\nu / \kappa$. These integrals can be evaluated by standard techniques; details of the calculation are available from the first listed author.

The effect of vortex wandering is to broaden the profiles and reduce the magnitude of the variations. In figure 3 we show values of the ratios $\bar{r}_{1} / r_{1}$ and $\bar{v}_{1} / v_{1}$ as functions of $\nu / \kappa$. We define $\bar{r}_{1}$ as the value of $r$ for which $\bar{v}$ attains its maximum $\bar{v}_{1}$. We also show the contributions $\overline{\Delta U_{v}} / \Delta U_{v}$ and $\overline{\Delta U_{\delta}} / \Delta U_{\delta}$ to the axial velocity on the mean centre-line. 


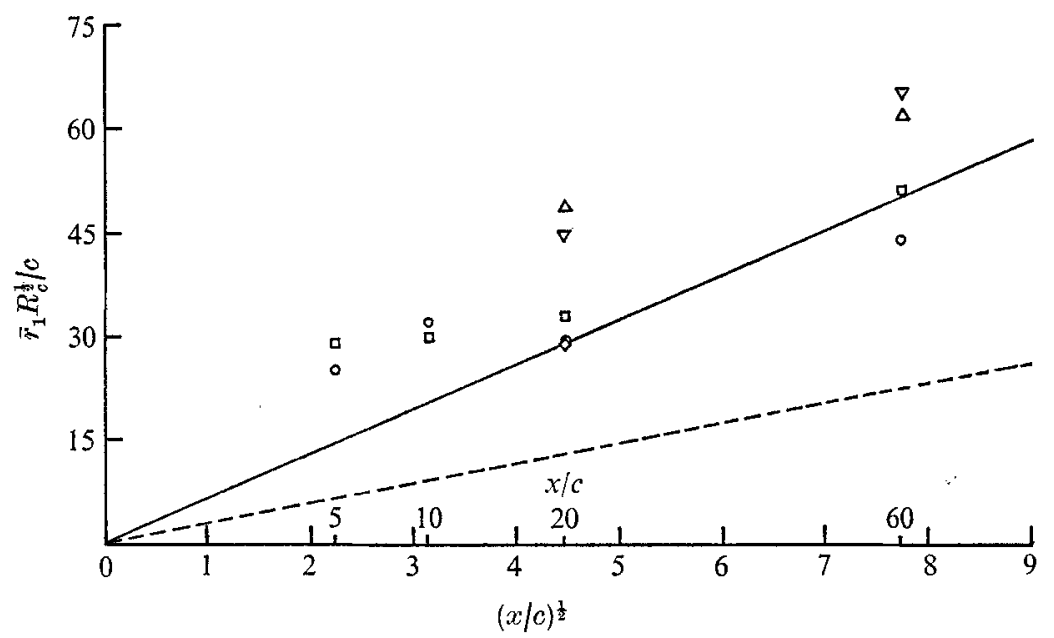

Figure 4. Dimensionless core radius as function of downstream distance. ----, instantaneous values [equation (3)]; ——, predictions averaged over vortex wandering for $\nu / \kappa=0 \cdot 25$. Model 1, square-tip planform; model 2, round-tip planform.

$\begin{array}{lccccc} & \bigcirc & \Delta & \nabla & \square & \diamond \\ \text { Model } & 1 & 1 & 1 & 2 & 2 \\ R_{0} \times 10^{-4} & 2 \cdot 2 & 7 \cdot 0 & 7 \cdot 0 & 2 \cdot 2 & 0 \cdot 75 \\ \alpha(\text { deg }) & 10 & 10 & 5 & 10 & 10\end{array}$

\subsection{Comparison with experiment}

We expect $\kappa$ to be a function of the tunnel Reynolds number, but since the estimate is rough, the refinement of employing different values for different tunnel speeds is uncalled for at the present stage. We have therefore used one typical value of the ratio $\nu / \kappa$, namely $0 \cdot 25$, in the comparisons between theory and experiment shown in figures 4-7. There does not seem to be any significant difference between the two model hydrofoils.

Figure 4 shows predicted and measured values of $\bar{r}_{1} R_{c}^{\frac{1}{2}} / c$, where $R_{c} \equiv U_{0} c / \nu$, as a function of $(x / c)^{\frac{1}{2}}$. Both the predicted values averaged over the vortex wandering (for $\nu / \kappa=0 \cdot 25$ ) and theinstantaneous predictions are shown. The experimental values of $\bar{r}_{1}$ are given by half the distance between the tangential velocity peaks. The agreement is reasonable in view of the experimental uncertainty in measuring $\vec{r}_{1}$ from data such as those shown in figure 2. In addition, the experiments show a lack of circular symmetry in the vortex structure, which is expected on theoretical grounds from detailed studies of the roll-up process (Saffman 1974), but cannot at present be incorporated in the analysis of the vortex structure given in $\mathrm{I}$. We emphasize that $\nu / \kappa$ was chosen on the basis of an independent measurement of the free-stream turbulence. The effect of vortex wandering upon the data is seen to be very significant.

Figure 5 shows $\bar{v}_{1} R_{c}^{-\frac{1}{4}} / U_{0} \alpha$ as a function of $(x / c)^{-\frac{1}{4}}$. The estimate of equation (2) for $\beta$ was employed. Both values averaged over the vortex wandering and instantaneous values are shown. The agreement is again reasonable except that there is a tendency for the theory to overestimate the velocity and axial vorticity, particularly for small values of $x / c$. In this connexion, it should be kept in mind 


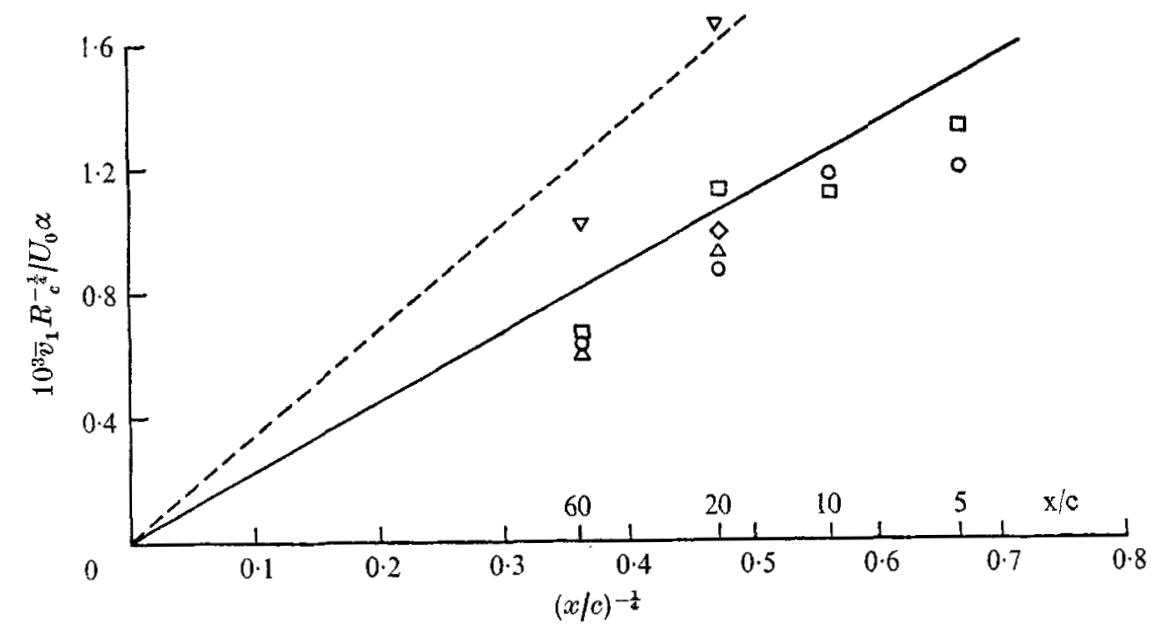

FIgURE 5. Dimensionless maximum tangential velocity velocity vs. downstream distance. - ---, instantaneous values; - $\longrightarrow$, predictions averaged over vortex wandering. $\alpha$ measured in degrees. Symbols as in figure 4.

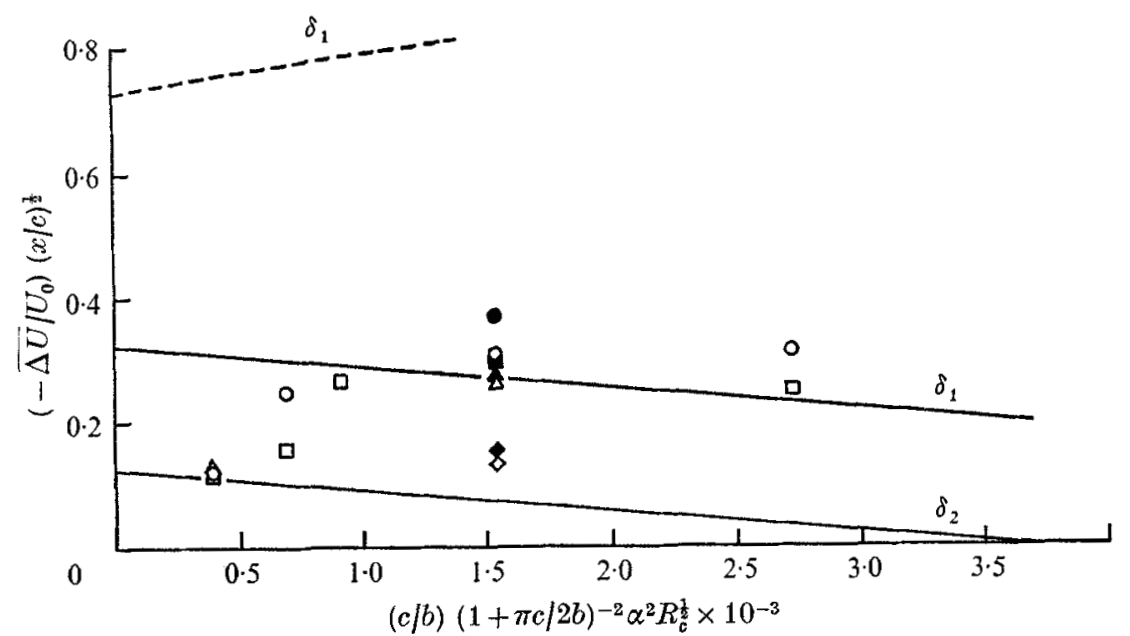

Figure 6. Axial velocity defect on mean axis as function of angle of attack, chord Reynolds number and aspect ratio. ----, instantaneous values (equations (7) and (2), using displacement thickness); - - predictions averaged over vortex wandering for $\nu / \kappa=0 \cdot 25$ using $\delta_{1}$ and $\delta_{2}$. Intercepts are at $\alpha^{2} R_{c}^{\frac{1}{c}}=8.9 \times 10^{5}$ and $3.5 \times 10^{5}$ for an aspect ratio of 6.18 . $\alpha$ measured in degrees.

\section{Model 1 \\ Model 2 \\ $x / c$}

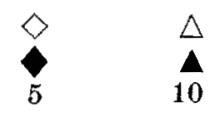

$\underset{10}{\Delta}$

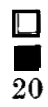

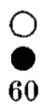

that the theory is an approximation formally valid in the limit of light loading and/or large downstream distances. Radial velocities in the cores are neglected, and these will be most important close to the wing. Also, our choice of $\beta$ is not free from uncertainty.

Figure 6 shows the axial velocity defect. We have plotted $\left(\overline{\Delta U} / U_{0}\right)(x / c)^{\frac{1}{2}} v s$. $\alpha^{2} R_{c}^{\frac{1}{2}}(c / b)(1+0 \cdot 5 \pi c / b)^{-2}$. There is some arbitrariness in the choice of $\delta$. It appears 


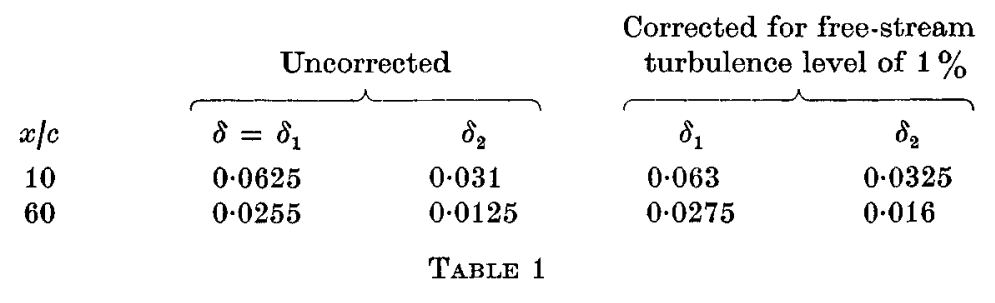

that $\delta_{1}$ is marginally better than $\delta_{2}$. The instantaneous value of $\Delta U$ is shown with $\delta_{1}$. The agreement is encouraging, and demonstrates again the importance of allowing for vortex wandering in the interpretation of experimental data. In particular, the slope of the theoretical line is reversed, and excess average velocities are predicted at the centre of the vortex when $\alpha^{2} R_{c}^{\frac{1}{2}} \geqslant 8.9 \times 10^{4}$, using $\delta_{1}$ for the boundary-layer retardation with $b / c=6 \cdot 18$. In this flow there would be a largeinstantaneous axial velocity deficit as measured, say, by flow visualization techniques. The data from figure 4 of Orloff \& Grant (1973) fall on the extrapolation of the curve representing averages over the vortex wandering shown in our figure 6 , using $\delta_{1}$, for abscissa values of about 100 . The value of $\nu / \kappa$ of $0 \cdot 25$ determined in the present experiment leads also to predictions of tangential velocity consistent with the data of Orloff \& Grant's figure 3.

Finally, we have computed the apparent turbulence intensity of the axial velocity due to vortex wandering. We calculated

$$
\overline{u^{2}(r, x)}=\iint\left\{u\left(\left[(r-\eta)^{2}+\zeta^{2}\right]^{\frac{1}{2}}, x\right)\right\}^{2} p(\eta, \zeta) d \eta d \zeta
$$

using the profile of $(6)$ in the integrand. Then the apparent turbulence intensity is

$$
\left(\overline{u^{\prime 2}}\right)^{\frac{1}{2}} / U_{0}=\left\{\overline{u^{2}(r, x)}-[\bar{u}(r, x)]^{2}\right\}^{\frac{1}{2}} / U_{0} .
$$

The predicted turbulence levels on the mean axis are shown in table 1 for the experimental case of figure 2. The second set of values in table 1 has been corrected for a free-stream turbulence level of $1 \%$, assuming statistical independence.

Since the measured values of the turbulence level shown in figure 2 do not seem to depend upon $x / c$ and the theoretical predictions behave as $(x / c)^{-\frac{1}{2}}$, there cannot be complete agreement. However, since the theory is likely to be more valid for $x / c=60$, we see that the predicted value there of 0.0275 using $\delta_{1}$ is close to the experimentally measured value.

The authors are indebted to the U.S. Air Force Office of Scientific Research for their support of this work. 


\section{REFERENCES}

Abramovitz, M. \& Stegun, T. A. 1965 Handbook of Mathematical Functions. Dover.

Adriax, R. 1972 J. Phys. E, Sci. Instrum. 5, 91.

CAIGER, B. \& GovLd, D. 1971 An analysis of flight measurements in the wake of a jet transport aircraft. In Aircraft Wake Turbulence and its Detection (ed. Olsen), pp. 125136. Plenum.

Chevalier, H. 1973 J. Aircraft, 10, 14.

GEORGE, W. 1972 Limitations on the measurement of unsteady flow velocities with a laser doppler velocimeter. In Proc. DISA Conf. on Flow in Industrial \& Medical Environments (ed. Cockrell), pp. 88-99. Leicester University Press.

Goldstein, R. 1967 Phys. Fluids, 10, 1349.

KNaPP, R. \& LEvY, J. 1948 The hydrodynamics laboratory of the California Institute of Technology. Trans. A.S.M.E., p. 437.

LEzIUS, D. 1973 Study of the far wake vortex field generated by a rectangular airfoil in a water tank. A.I.A.A. Paper, no. 73-682.

Mason, H. \& Marchman, J. 1972 The farfield structure of aircraft wake turbulence. A.I.A.A. Paper, no. 72-40.

MTLLER, E. \& BRown, C. 1971 An experimental study of trailing vortex wakes using a large towing tank. HYDRONAUTICS Tech. Rep. no. 7105-1.

Moore, D. W. \& Saffman, P. G. 1973 Proc. Roy. Soc. A 333, 491-508.

OLSEN, J.H. 1971 Results of trailing vortex studies in a towing tank. In Aircraft Wake Turbulence and its Detection, pp. 455-472. Plenum.

OrLoff, K. \& Grant, G. 1973 The application of a scanning laser-doppler velocimeter to trailing vortex definition and alleviation. A.I.A.A. Paper, no. 73-680.

Safrman, P. G. 1974 The structure and decay of trailing vortices. (To be published.)

WANG, C. 1972 Appl. Phys. Lett. 20, 339. 